\title{
Improving the volumetric specific capacitance of flexible polyaniline electrode: solution casting method and effect of reduced graphene oxide sheets
}

\author{
Jingjing Wang ${ }^{1}$, Weihua $\mathrm{Su}^{1}$, Jinmeng Zhang ${ }^{1}$, An'an Zhou ${ }^{1}, \mathrm{Hua} \mathrm{Bai}^{{ }^{*}}$ and Tongyi Zhang ${ }^{{ }^{*}}$
}

\begin{abstract}
High volumetric specific capacitance is essential for flexible supercapacitors. In this paper, a flexible composite electrode with ultrahigh volumetric specific capacitance and good rate performance is designed and prepared. The film electrode is composed of non-porous compact polyaniline as the matrix and a small amount of reduced graphene oxide sheets as the conductive filler. The film is prepared by a newly developed solution casting method, where the casting solution is obtained by self-assembly of polyaniline and graphene oxide in a blended solution. A systematical investigation on the effect of reduced graphene oxide sheets reveals that they serve as both the conductive filler and the diffusion barrier. With the optimized reduced graphene oxide content, a large volumetric specific capacitance of $1354 \mathrm{~F} \mathrm{~cm}^{-3}$ at $2.4 \mathrm{~A} \mathrm{~cm}^{-3}$ is achieved, and good rate performance is also obtained because of the good ionic conductivity of polyaniline. This work provides a high-performance electrode for flexible supercapacitors, and the solution casting method is also valuable in fabricating other organic electrode materials.
\end{abstract}

Keywords: conducting polymer, graphene, compact film, conductivity, diffusion barrier

\section{INTRODUCTION}

Flexible energy storage devices are highly demanded with the rapid development of wearable electronic devices [14]. Flexible supercapacitors are an important group of flexible energy storage devices due to their high rate performance and long cycling life [5-7]. Considering the practical application, volumetric specific capacitance is essential for flexible supercapacitors $[8,9]$. Generally, the volumetric specific capacitance of an electrode material can be calculated from its gravimetric specific capacitance and apparent density. For an electrode material with a certain composition, the main method of promoting its volumetric specific capacitance is to increase the apparent density, for example, by reducing the porosity. However, the interconnected pores inside the electrode serve as a "high way" for electrolyte diffusion and reducing the porosity of the electrode usually leads to a decrease in the rate performance. Therefore, it requires accurate control of the porosity and microstructure of the electrode materials to achieve a high volumetric specific capacitance at large current density.

At present, carbon materials, conducting polymers and transition metal oxides are common electrode materials in supercapacitors [10-12]. Among these materials, polyaniline/reduced graphene oxide (PANI/RGO) composites have attracted considerable attention because PANI provides large pseudocapacitance via redox reactions and RGO sheets form conducting path for electron transport [13-16]. More importantly, as a hydrophilic polymer, PANI is highly solvated in the electrolyte and thus has large ion conductivity, which allows the PANIbased electrode to possibly maintain a high rate performance even at low porosity [17-20]. Conventional methods of fabricating the PANI-based film electrode are mainly based on in-situ polymerization of aniline on a flexible matrix. For example, in-situ polymerization in hydrogel yields flexible and porous PANI composite hydrogel $[21,22]$. However, these methods are designed for depositing PANI into a porous matrix or onto the surface of the substrate. They are not suitable for preparing a high-density PANI/RGO composite with low porosity and high volumetric specific capacitance. Also, by the insitu polymerization, it is difficult to control the compo-

\footnotetext{
${ }^{1}$ College of Materials, Xiamen University, Xiamen 361005, China

${ }^{2}$ Materials Genome Institute, Shanghai University, Shanghai 200444, China

* Corresponding authors (emails: baihua@xmu.edu.cn (Bai H); Zhangty@shu.edu.cn (Zhang T))
} 
sition of the prepared PANI/RGO electrode. Obviously, a novel method is desirable to develop PANI/RGO electrode materials with high volumetric specific capacitance.

In this paper, we fabricated flexible PANI/RGO composite films with an ultrahigh volumetric specific capacitance, by using a newly developed solution casting method. Stable solutions of PANI/GO are obtained by self-assembling of PANI and graphene oxide (GO) in a blended solvent of $N$-methyl-2-pyrrolidone (NMP) and water, and they are cast into large-area, uniform and nonporous PANI/RGO films with high apparent density. This method also allows us to accurately control the content of RGO over a wide range (0-100\%) and systematically study the effect of RGO on the capacitive performance. Our results confirm that besides as the conductive filler to enhance the mechanical strength, RGO sheets also play a role of diffusion barriers to the electrolyte. Systematical investigation finds that $10 \mathrm{wt} . \%$ RGO in composite films leads to the highest volumetric specific capacitance of $1354 \mathrm{~F} \mathrm{~cm}^{-3}$ and good rate performance.

\section{EXPERIMENTAL SECTION}

\section{Materials}

Natural graphite powder (325 mesh) was bought from Qingdao Huatai Lubricant Sealing S\&T Co. Ltd. Potassium permanganate (AR), sodium nitrate (AR), hydrogen peroxide $(30 \%)$, sulfuric acid $\left(\mathrm{H}_{2} \mathrm{SO}_{4}, 98 \%\right)$, hydrochloric acid ( $\mathrm{HCl}, \mathrm{AR})$, aqueous ammonia $(28 \%)$, and ammonium persulfate (AR) were purchased from XiLong Scientific Co., Ltd. Aniline and 1-methyl-2-pyrrolidinone (AR) were the products of Aladdin Industry Co., Ltd. All the chemicals were used as received without further purification. GO was prepared by oxidation of natural graphite powder according to a modified Hummers' method [23]. PANI was synthesized by chemical oxidation polymerization [24], and then converted into the emeraldine base state by ammonia treatment to increase its solubility in NMP. The dried PANI powder was dissolved in NMP by agitation at room temperature, and the obtained solution was filtered, giving a PANI stock solution of $\sim 37.1 \mathrm{mg} \mathrm{mL}^{-1}$.

\section{Preparation of PANI/RGO composite films}

$\mathrm{PG}_{x}$ denotes a PANI/RGO composite film with $x$ representing the weight percentage of GO over PANI+GO in the sample preparation. In order to prepare $\mathrm{PG}_{30}$, $0.566 \mathrm{~mL}$ of PANI stock solution $\left(37.1 \mathrm{mg} \mathrm{mL}^{-1}\right)$ was diluted by adding $28.375 \mathrm{~mL}$ of NMP, and then $1.059 \mathrm{~mL}$ of $\mathrm{GO}$ aqueous dispersion $\left(9.4 \mathrm{mg} \mathrm{mL}^{-1}\right)$ was added into the above PANI solution under violent agitation. The produced homogeneously mixed dispersion was poured into a ceramic or poly(tetrafluoroethylene) (PTFE) mold, and dried at $75^{\circ} \mathrm{C}$ in air for $8 \mathrm{~h}$, yielding a free-standing PANI/GO film. The film was chemically reduced by soaking in hydroiodic acid (45\%) for $1 \mathrm{~h}$ and repeatedly rinsing with ethanol and water to remove residual iodine. After being dried at room temperature, $\mathrm{PG}_{30}$ film was prepared. To prepare other PANI/RGO films, the volume of PANI solution and GO dispersion was adjusted to the corresponding values. The RGO film was prepared following the same procedure, except that only GO aqueous solution was used in the casting. Pure PANI film was obtained by directly drying the PANI solution in NMP at $75^{\circ} \mathrm{C}$.

\section{Fabrication of flexible solid-state device}

The poly(vinyl alcohol) (PVA)/ $\mathrm{H}_{3} \mathrm{PO}_{4}$ gel electrolyte was prepared as follows: $6 \mathrm{~g}$ of PVA powder was added in $60 \mathrm{~mL}$ of deionized water and heated to $95^{\circ} \mathrm{C}$ under agitation, until the solution became clear. After the solution cooled down, $3.2 \mathrm{~mL}$ of phosphoric acid was added. The mixed solution was spread onto a culture dish and dried at $50^{\circ} \mathrm{C}$ for $5 \mathrm{~h}$ to form a PVA/ $\mathrm{H}_{3} \mathrm{PO}_{4}$ gel film [25]. The $\mathrm{PVA} / \mathrm{H}_{2} \mathrm{SO}_{4}$ gel electrolyte was prepared by dissolving $6 \mathrm{~g}$ of PVA powder and $3.28 \mathrm{~mL}$ of $\mathrm{H}_{2} \mathrm{SO}_{4}$ in $60 \mathrm{~mL}$ of deionized water at $95^{\circ} \mathrm{C}$ [26]. The $\mathrm{PG}_{10}$ film was cut into two rectangular pieces $(1 \mathrm{~cm} \times 1.5 \mathrm{~cm})$ and put on the gold-coated polyimide substrate. The $\mathrm{PVA} / \mathrm{H}_{2} \mathrm{SO}_{4}$ gel electrolyte aqueous solution was slowly dropped onto the $\mathrm{PG}_{10}$ films and dried in the ambient environment for $12 \mathrm{~h}$. Then the two electrodes were pressed together with $\mathrm{PVA} / \mathrm{H}_{3} \mathrm{PO}_{4}$ gel electrolyte film as a separating membrane between two electrodes.

\section{Electrochemical measurements}

The electrochemical performance of the PANI/RGO composite film was tested in a three-electrode system. $1 \mathrm{~mol} \mathrm{~L}^{-1} \mathrm{H}_{2} \mathrm{SO}_{4}$ was used as the electrolyte and Pt foil was used as a current collector. The counter electrode was an RGO hydrogel [27] and the reference electrode was an saturated calomel electrode (SCE). The PANI/RGO composite film was cut into a $1.5 \mathrm{~cm} \times 1.5 \mathrm{~cm}$ square as the working electrode. The film was immersed in the electrolyte solution for $12 \mathrm{~h}$ before the test to allow the complete infiltration of electrolyte into the electrode materials. The electrochemical impedance spectroscopy (EIS) was recorded under their open circuit potential. The specific capacitance of the film was calculated by galvanostatic charge discharge (GCD) tests using the equation: 
$C=I \Delta t / K(\Delta U-I R)$,

where $I$ is the constant discharge current, $t$ is the discharging time, $K$ is the mass (for gravimetric specific capacitance) or the volume (for volumetric specific capacitance) of the composite film, $U$ is the potential range of the working electrode (from 0 to $0.8 \mathrm{~V}$ ), and $I R$ is the voltage drop upon discharging.

The specific capacitance of the film in a flexible device was calculated by GCD tests using the equation:

$C=I \Delta t / K\left(U_{\mathrm{M}}-I R\right)$,

where $K$ is the total mass (for gravimetric specific capacitance) or the volume (for volumetric specific capacitance) of two electrodes, $U_{\mathrm{M}}$ is the highest working voltage of the device.

The volumetric energy density of the electrode material in the flexible device was determined by the following equation:

$E=I / V \int_{\text {discharge }} U \mathrm{~d} t$

where $V$ is the total volume of the two electrodes, $U$ is the voltage of the device. The volume of the film electrode was calculated from the area and the thickness, which was determined by averaging the thicknesses measured from the cross-sectional SEM image at different positions. The volumetric power density $(P)$ was calculated by:

$P=E / \Delta t$,

where $t$ is the corresponding discharge time.

\section{Characterization}

All the electrochemical measurements were performed on a $660 \mathrm{E}$ electrochemical workstation (CHI, China). The morphology of the composite films was observed on a SU-70 field emission scanning electron microscope (SEM, Hitachi, Japan) at $10 \mathrm{kV}$. All the samples were sputtered with an Au layer for SEM observation. Raman spectra were collected by using XPLORA Raman spectrometer (Horiba, Japan) with a 532-nm laser as a light source. The $\mathrm{X}$-ray diffraction (XRD) was measured on a D8 X-ray diffractometer (Bruker, USA) with $\mathrm{Cu} \mathrm{Ka}$ source. X-ray photoelectron spectroscopy (XPS) was collected on the Escalab 250Xi X-ray photoelectron spectroscopy (Thermo Fisher, USA) using monochromated X-rays from an $\mathrm{Al}$ $\mathrm{Ka}$ source. The conductivity was tested on a dual-electric test four-probe conductivity meter (Guangzhou fourprobe electronic, China).

\section{RESULTS AND DISCUSSION}

Fig. 1a shows the solution casting method, where a blended solution of PANI/GO in NMP/water was obtained by mixing the solution of PANI in NMP and the GO aqueous dispersion. GO is a two-dimensional (2D) surfactant and soluble in both water and NMP. PANI chains can self-assemble onto the GO sheets via $\pi-\pi$ stacking and hydrogen bond. Meanwhile, PANI in the emeraldine base state is partially doped by the carboxyl groups on GO, and forms electrostatic interaction with GO. Therefore, PANI and GO were dispersed homogeneously in the mixed solvent and the solution homogeneity was stable over several weeks (Fig. S1). The blend solution was cast and dried on a PTFE or alumina ceramics substrate, producing a uniform PANI/GO film, which was then reduced by hydroiodic acid to give a pristine PANI/RGO film. During the reduction process, PANI in the emeraldine base was also doped by hydroiodic acid, and thereby the final product is emeraldine salt/RGO composite film.

To optimize the capacitance performance of PANI/ RGO films, the content of GO in the composite was controlled from 0 to 100 wt.\%, and its effect on the properties of PANI/RGO composite films was investigated systematically. With the novel casting method, it is easy to fabricate large-area PANI/RGO film as long as a large casting mold is available. Here a $900-\mathrm{cm}^{2} \mathrm{PG}_{30}$ film is demonstrated in Fig. 1b, and the large-area film is expected to provide more possibilities in the practical applications.

Fig. 1c-j and Figs S2-S4 are the SEM images of the asprepared PANI/GO and PANI/RGO films. The corresponding PANI/GO and PANI/RGO films have similar morphology, and thus the hydroiodic acid does not change the morphology and structure of the films. All the PANI/RGO films have a compact and smooth surface. Films with high RGO contents (Fig. 1c, d) have wrinkles on their surfaces, which is the characteristic of RGO. As the RGO content decreases (Fig. 1e, f), the wrinkles on the surface become less, and only small grains appear, which are the typical surface feature of a polymer film. In the cross-sectional view (Fig. 1g-j), the lamellar structure was observed for all the films, even when the PANI content was as high as $90 \mathrm{wt} . \%$. The similar lamellar structures were also found in other RGO/polymer composite films [28], attributed to the lyotropic liquid crystal property of GO sheets. These lamellar structures can efficiently enhance the mechanical strength of the composite film [29-31]. No PANI aggregates or/and pores were detected in the SEM observations. The nitrogen, carbon, and oxygen element signals were homogeneous in the energy-dispersive X-ray spectroscopy (EDS) mapping of 
a

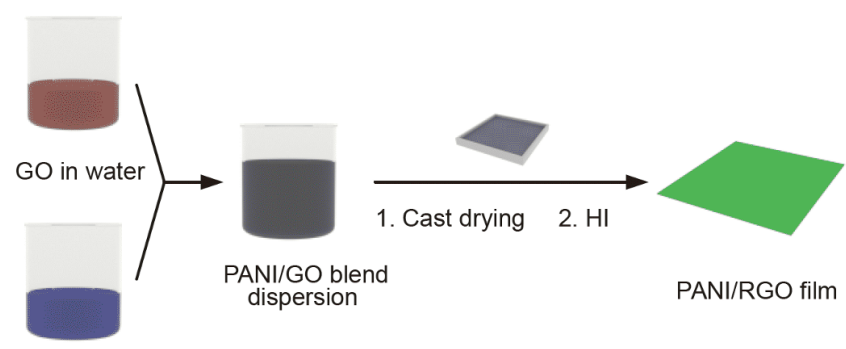

PANI in NMP b

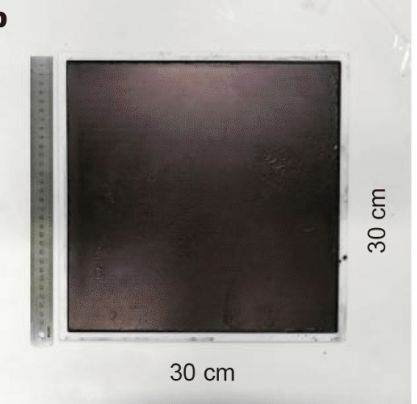

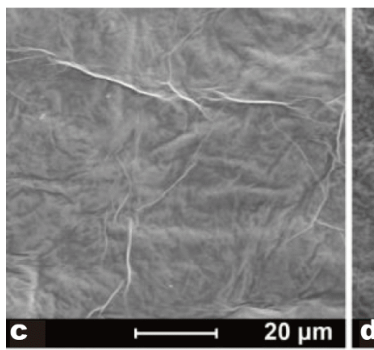
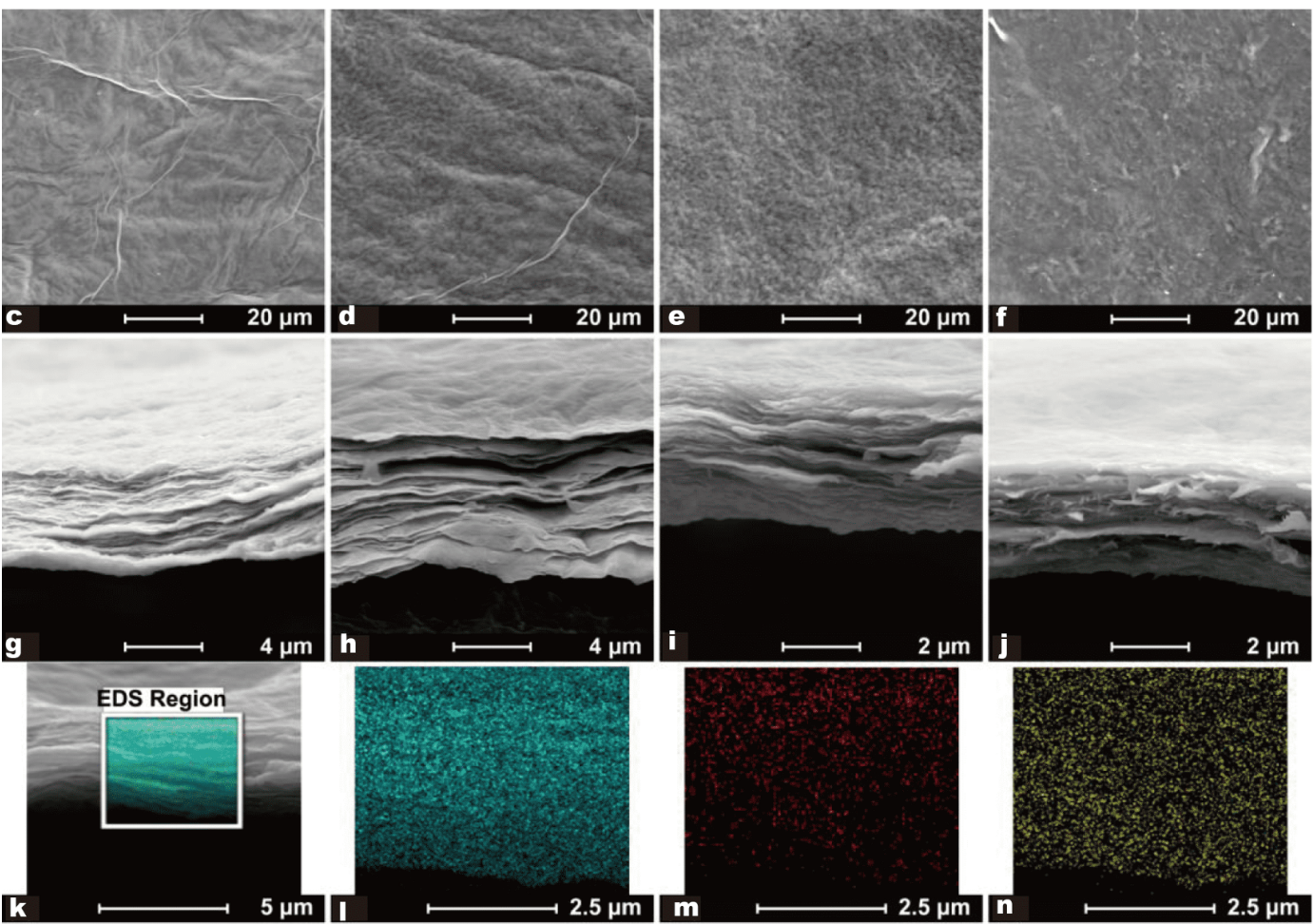

Figure 1 Preparation and morphology of the PANI/RGO composite films. (a) Schematic illustration of the preparation procedure (HI represents hydroiodic acid). (b) Photograph of a 900-cm ${ }^{2}$ PANI/RGO film. (c-f) SEM images of the top surface view of RGO (c), $P_{90}(\mathrm{~d}), \mathrm{PG}_{50}$ (e), and PG 10 (f). $(\mathrm{g}-\mathrm{j})$ SEM images of the cross-sectional view of RGO (g), $\mathrm{PG}_{90}(\mathrm{~h}), \mathrm{PG}_{50}(\mathrm{i})$, and $\mathrm{PG}_{10}(\mathrm{j})$. (k-n) EDS mapping of $\mathrm{C}(\mathrm{l})$, $\mathrm{N}(\mathrm{m})$, and $\mathrm{O}(\mathrm{n})$ elements on the cross-section of $\mathrm{PG}_{50}$.

the cross-section of the film (Fig. 1k-n), suggesting a uniform distribution of PANI and RGO in the film. It is difficult for conventional in-situ polymerization method to produce a uniform and free-stranding PANI/RGO composite film with very high PANI content, while the novel casting method can easily achieve the high uniformity, which improves the electrochemical energy storage performance.

Fig. 2a shows the $P_{30}$ survey XPS spectrum, indicating the iodine $3 \mathrm{~d}_{5 / 2}$ peak at $617 \mathrm{eV}$ and iodine $3 \mathrm{~d}_{3 / 2}$ at $629 \mathrm{eV}$ [32]. This iodine can be attributed to the iodide ions, which are the counter ions of the doped PANI. The deconvolution of $\mathrm{PG}_{30} \mathrm{C}$ 1s lines (Fig. 2b) show the bonds of
$\mathrm{C}-\mathrm{C} / \mathrm{C}=\mathrm{C}(284.6 \mathrm{eV}), \mathrm{C}-\mathrm{O}(285.2 \mathrm{eV}), \mathrm{C}-\mathrm{O}(286.1 \mathrm{eV})$, $\mathrm{C}=\mathrm{O}(287.3 \mathrm{eV})$, and $\mathrm{O}-\mathrm{C}=\mathrm{O}(289.9 \mathrm{eV})[33]$, where the intensity of $\mathrm{C}-\mathrm{O}$ and $\mathrm{C}=\mathrm{O}$ peaks from the $\mathrm{PG}_{30}$ film is much lower than that in the corresponding PANI/GO film, indicating GO is successfully reduced by hydroiodic acid. The structure of the composite film was further investigated by XRD. As shown in Fig. 2c, RGO film has a broad diffraction peak around $24.5^{\circ}$, corresponding to the interlayer spacing of $0.36 \mathrm{~nm}$ of the RGO sheets [34]. As the PANI content increases, this peak becomes weaker, showing that PANI is intercalated between the RGO. The XRD patterns confirm that the RGO sheets and PANI are uniformly distributed. For all the films, the absence of 

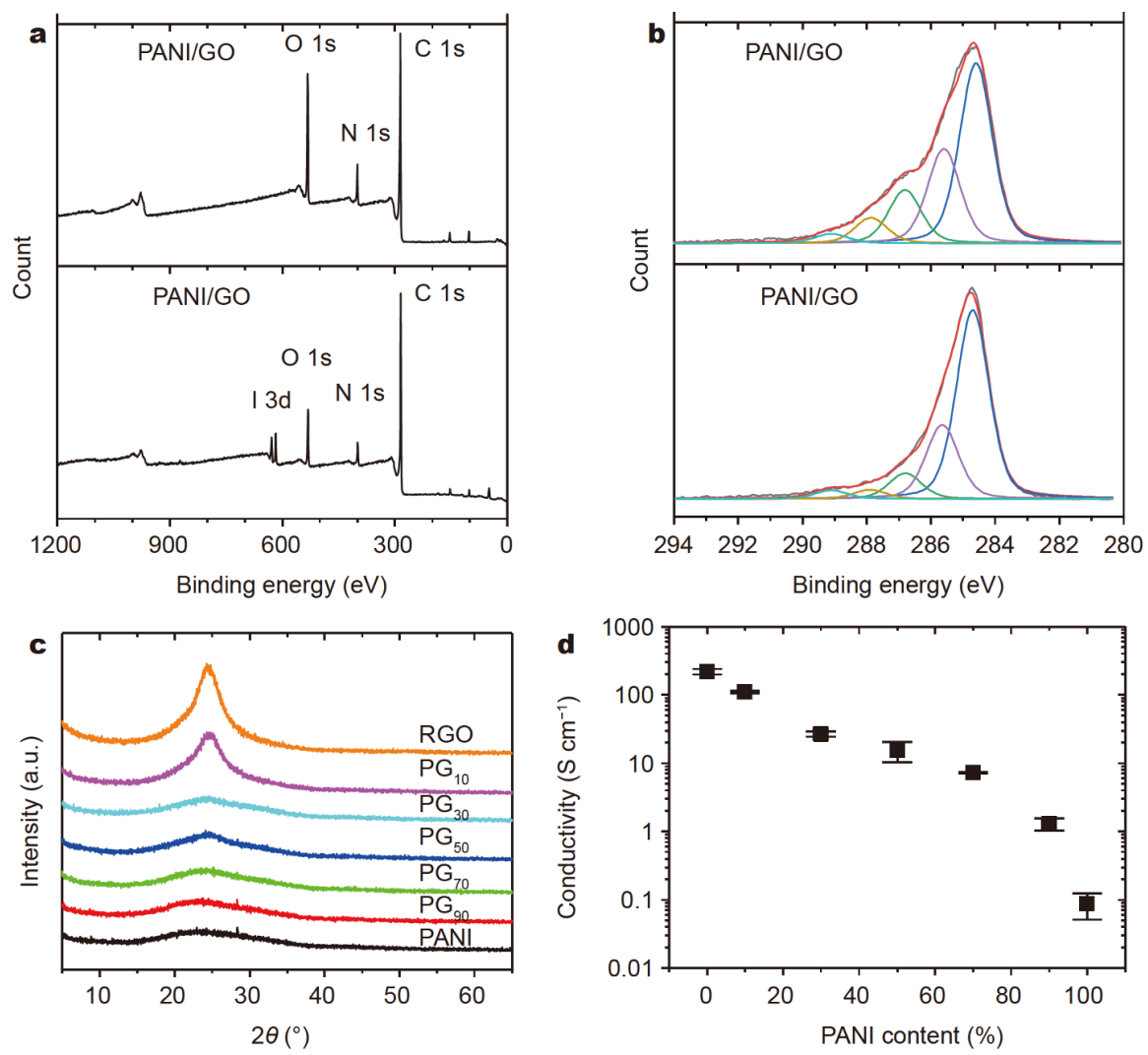

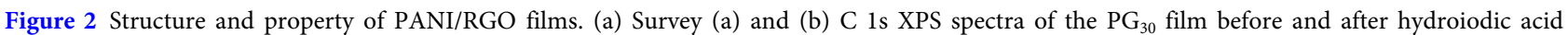
reduction. (c) XRD patterns of RGO, PANI, and PANI/RGO films. (d) Electric conductivities of PANI/RGO, RGO, and PANI film.

diffraction peak associated with GO $\left(\sim 11^{\circ}\right)$ again reveals the complete reduction of GO. Raman spectra of the composite films (Fig. S5) also show the characteristic bands of the doped PANI. In summary, all the results confirm the successful preparation of uniform PANI/ RGO films.

Electric conductivities of the PANI/RGO composites were measured to evaluate the reduction level of RGO and the charge transport ability of the composite. As shown in Fig. 2d, the pure PANI film has a small conductivity of $8.7 \times 10^{-2} \mathrm{~S} \mathrm{~cm}^{-1}$, but for the $\mathrm{PG}_{10}(10 \mathrm{wt} . \%$ GO feeding ratio), the conductivity increases to $1.3 \mathrm{~S} \mathrm{~cm}^{-1}$. The improvement in film conductivity further confirms the successful reduction of GO by hydroiodic acid [35]. The conductivity of PANI/RGO film increases with the RGO content, and reaches $109.4 \mathrm{~S} \mathrm{~cm}^{-1}$ for $\mathrm{PG}_{90}$. Although high RGO content increases conductivity, high RGO content decreases the specific capacitance of the composite film due to a decrease in the PANI content. Therefore, the RGO content should be optimized for practical application.

The electrochemical performance of the $\mathrm{PG}_{x}$ films was tested in a three-electrode cell. Fig. S6 shows the cyclic voltammetric $(\mathrm{CV})$ curves of pure PANI film, pure RGO film, and PANI/RGO composite films with a scanning rate of $50 \mathrm{mV} \mathrm{s}^{-1}$ at a potential range of $-0.2-0.8 \mathrm{~V} v s$. SCE (all the potential was referenced to SCE unless otherwise stated). The CV curve of the pure RGO film has a rectangular shape, showing the characteristics of electric double-layer capacitance. The CV curves of the PANI/ RGO composite films show a pair of redox peaks at $\sim 0.5 \mathrm{~V}$, corresponding to the redox of oligoanilines [36], as indicated by Fig. 3a. The oligoanilines are typical electrochemical decomposition products of PANI, but they have larger specific capacitance than PANI. Fig. 3b shows the GCD curves of the PANI/RGO films measured under the preset maximum potential of $0.8 \mathrm{~V}$ at the current density around $1 \mathrm{~A} \mathrm{~g}^{-1}$ (see Figs S7-S13 for GCD curves at other current densities). The plateau at $>0.7 \mathrm{~V}$ in the charge curve indicates slight over-oxidation of PANI, which generates oligoaniline [36]. The shoulder in each discharging curve of the PANI/RGO composite films between $0.4-0.5 \mathrm{~V}$ is attributed to the redox of oligoaniline. 
$\mathbf{a}$
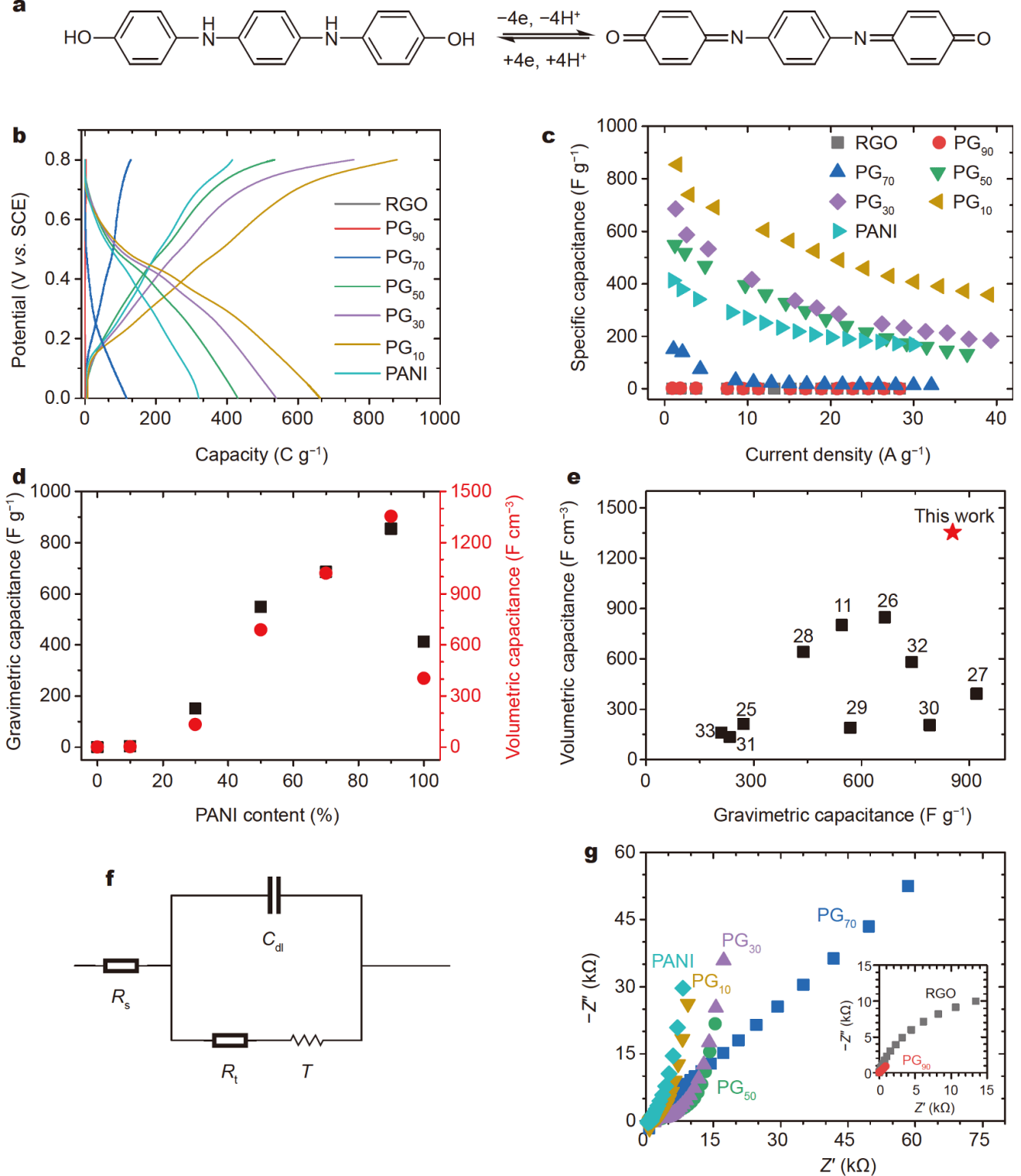

Figure 3 Electrochemical performance of RGO, PANI, and PANI/RGO composite films. (a) Typical redox reaction of oligoaniline. (b) The GCD curves. (c) Volumetric specific capacitances at different current densities. (d) Gravimetric specific capacitances and volumetric specific capacitances of the films (current density: $1 \pm 0.5 \mathrm{~A} \mathrm{~g}^{-1}$ ). (e) The comparison of volumetric specific capacitance of $\mathrm{PG}_{10}$ and materials in literature. (f) Equivalent circuit of the PANI/RGO electrode. (g) Nyquist plots of the electrodes.

The gravimetric specific capacitance was calculated according to the GCD curve and depicted in Fig. 3c. The pure RGO film has a tiny specific capacitance of $0.3 \mathrm{~F} \mathrm{~g}^{-1}$ at $0.94 \mathrm{~A} \mathrm{~g}^{-1}$. The specific capacitance of the composite films increases as the content of PANI increases (Fig. 3d), and reaches a maximum at a $90 \mathrm{wt} . \%$ PANI content. For $\mathrm{PG}_{10}$, the gravimetric specific capacitance reaches $854 \mathrm{~F} \mathrm{~g}^{-1}$ at $1.5 \mathrm{~A} \mathrm{~g}^{-1}$, and at a high current density of $39.0 \mathrm{~A} \mathrm{~g}^{-1}$, the gravimetric specific capacitance is still
$356 \mathrm{Fg}^{-1}$. These values are among the highest specific capacitances of the flexible PANI-based composites. The previous study [36] confirmed that the oligoaniline produced by electrochemical degradation of PANI could contribute large pseudocapacitance, but must be with the assistance of a good electric conductive matrix. Here the RGO sheets serve as the electron conductive path in the composite and $10 \mathrm{wt} . \%$ RGO in the $\mathrm{PG}_{10}$ composite increases significantly the capacitance to $854 \mathrm{~F} \mathrm{~g}^{-1}$. 
Because the films have a non-porous compact structure, they exhibit high volumetric specific capacitances. Fig. 3d shows that the highest volumetric specific capacitance is $1354 \mathrm{~F} \mathrm{~cm}^{-3}$ at $2.4 \mathrm{~A} \mathrm{~cm}^{-3}$, and even at $62.4 \mathrm{~A} \mathrm{~cm}^{-3}$, the volumetric specific capacitance is still as high as $564 \mathrm{~F} \mathrm{~cm}^{-3}$. these values are much higher than other PANI-based electrode materials as shown in Fig. 3e [18,37-45]. It has been reported that PANI has a high ionic conductivity, which allows the interior of dense films to participate in electrochemical reactions. Therefore, we can use our solution-casting method to produce the compact composite film with high density, and improve the volumetric specific capacitance. However, it should be noted that although RGO sheets can improve the electric conductivity and specific capacitance of the PANI/RGO films, they may hinder the diffusion of electrolytes. This is confirmed by the EIS results (Fig. 3f, g). An equivalent circuit of the PANI/RGO electrode is shown in Fig. $3 \mathrm{f}$. $R_{\mathrm{s}}$ is the series resistance of the current collector and electrolyte, $C_{\mathrm{dl}}$ is the electric double layer capacitance, $R_{\mathrm{t}}$ is the charge transfer resistance of the PANI redox, and $T$ is the finite space Warburg impedance. The finite space Warburg impedance, which gives a straight line with a phase of $45^{\circ}$ and an almost vertical line at the low-frequency region in the Nyquist plot, can be used to investigate the diffusion in the electrode. With the increase of the RGO content (Fig. 3g), the $45^{\circ}$-region becomes longer, corresponding to larger equivalent series resistance and a smaller diffusion coefficient of the electrolyte inside the film. While for $\mathrm{PG}_{70}$, $\mathrm{PG}_{90}$, and RGO film, the diffusion is so slow that the EIS does not show capacitive characteristics. In general, the RGO content can be easily controlled to achieve a balance between the mechanical strength, capacitance, and rate performance, to meet the requirements of different practical applications. In our experiments, $10 \mathrm{wt} . \%$ RGO sheets can efficiently increase the conductivity of the film, but do not reduce the diffusion rate significantly. Therefore, $\mathrm{PG}_{10}$ shows both high volumetric specific capacitance and good rate performance.

A symmetric solid-state flexible device was assembled by using the $\mathrm{PG}_{10}$ film as the electrodes and $\mathrm{H}_{2} \mathrm{SO}_{4}$-PVA hydrogel as the electrolyte. The nonlinear GCD curves of the device show the character of pseudocapacitance (Fig. 4a), and the specific capacitance was calculated to be $198 \mathrm{~F} \mathrm{~g}^{-1}$ at $0.83 \mathrm{~A} \mathrm{~g}^{-1}$, corresponding to an energy density of $15.1 \mathrm{~mW} \mathrm{~h} \mathrm{~cm}^{-3}$ and a power density of $0.34 \mathrm{~W} \mathrm{~cm}^{-3}$ (Fig. S14 for Ragone plot). The average single electrode specific capacitance was calculated to be $792 \mathrm{~F} \mathrm{~g}^{-1}$ at $1.7 \mathrm{~A} \mathrm{~g}^{-1}$. This value is slightly smaller than the three-electrode result because of the lower conductivity and weaker permeability of the gel electrolyte. When the current density increases to $4.4 \mathrm{~A} \mathrm{~g}^{-1}$, the specific capacitance still remains at $124 \mathrm{~F} \mathrm{~g}^{-1}$ (Fig. 4b), giving the volumetric energy density and power density of $1.0 \mathrm{~mW} \mathrm{~h} \mathrm{~cm}^{-3}$ and $0.53 \mathrm{~W} \mathrm{~cm}^{-3}$, respectively. These values are larger than those of most of the reported flexible supercapacitors [18,46-52]. Fig. S15 shows that when the supercapacitor was bent, the discharge time did not decrease, indicating good flexibility of the solid device. Fig. 4d and Movie S1 show that three series-connected devices, which have a working voltage of $2.4 \mathrm{~V}$, can light a
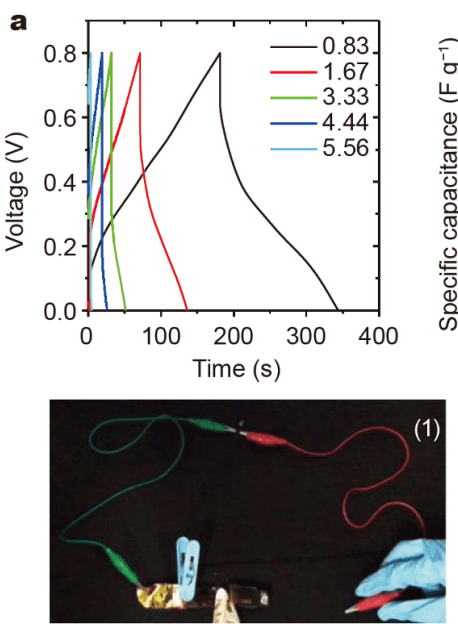
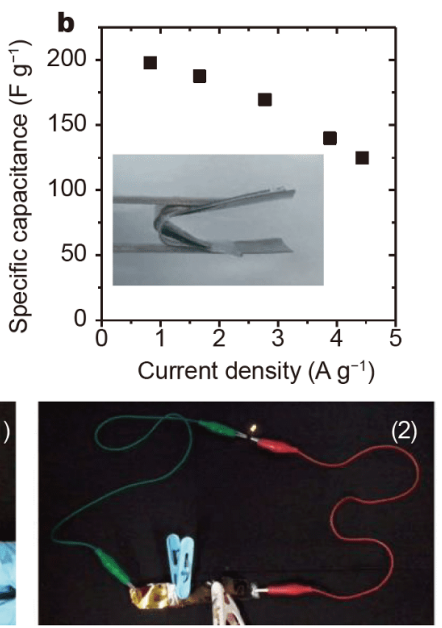
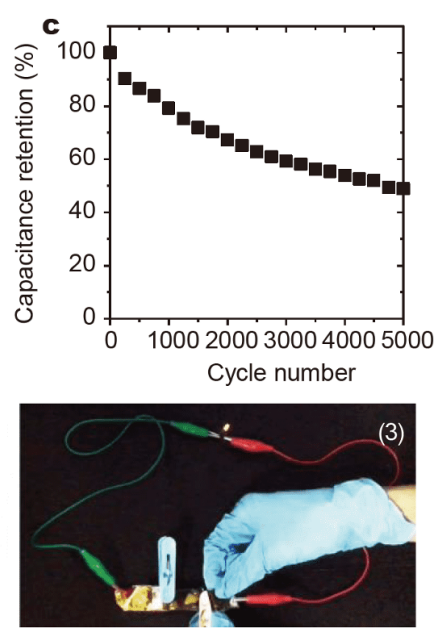

Figure 4 Electrochemical performance of the all-solid-state device. (a) The GCD curves at different current densities. (b) Rate performance of the device. Inset: photograph of a folded device. (c) Cycle stability of the device at $3.33 \mathrm{~A} \mathrm{~g}^{-1}$. (d) Photograph of an LED driven by three series-connected devices. (1) The LED before being connected to the supercapacitors. (2) The LED lighten by the supercapacitors. (3) The LED lighten by the bent supercapacitors. 
2-V LED. When the devices were bent, the brightness of the LED kept stable. After 5000 charge/discharge cycles, the capacitance droped by $50 \%$ (Fig. 4c), due to the electrochemical degradation of PANI in anode at high voltage. The above results demonstrate that the PANI/ RGO films are good flexible electrodes.

The mechanical strength of the electrode materials on the capacitive performance was investigated in the present study and such property has been seldom studied yet. The present study shows that RGO sheets significantly increase the shape stability of PANI during the electrochemical test. As a high-strength $2 \mathrm{D}$ material, RGO sheets are reported to efficiently increase the modulus and fracture strength of various polymers [53,54]. Fig. 5 shows that the pure PANI film became shattered after 10,000 cycles of charging/discharging tests, while the $P_{10}$ film maintained intact after 10,000 cycles. Clearly, composing PANI with RGO sheets makes the supercapacitor more mechanically reliable and gives it longer service life.

\section{CONCLUSIONS}

A solution casting technique was developed to fabricate flexible PANI/RGO composite films with compact PANI matrix and uniformly distributed RGO sheets, which produced large-area PANI/RGO films with precisely controlled RGO content. The RGO sheets increase the conductivity and mechanical strength of the films and the PANI provides high pseudocapacitance. Moreover, RGO sheets also play the roles of diffusion barriers to the electrolyte and mechanical reinforcer. The optimized PANI/RGO composite films containing 10\% RGO exhibit the highest volumetric specific capacitance of $1354 \mathrm{~F} \mathrm{~cm}^{-3}$ at $2.4 \mathrm{~A} \mathrm{~cm}^{-3}$. Accordingly, the flexible supercapacitors

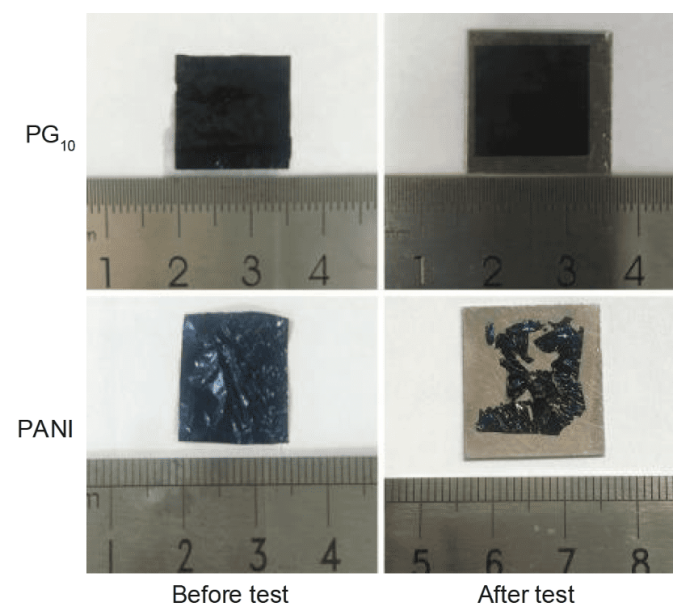

Figure 5 Photographs of $\mathrm{PANI}$ and $\mathrm{PG}_{10}$ films before and after the electrochemical test. with $\mathrm{H}_{2} \mathrm{SO}_{4}$-PVA electrolyte were fabricated and showed excellent performance. These results confirm that PANI/ RGO is a promising electrode material for high-density energy storage, and the solution casting method provides a new option of fabricating other organic electrode materials.

Received 20 May 2020; accepted 23 July 2020;

published online 13 October 2020

1 Wang X, Lu X, Liu B, et al. Flexible energy-storage devices: design consideration and recent progress. Adv Mater, 2014, 26: 47634782

2 Li L, Wu Z, Yuan S, et al. Advances and challenges for flexible energy storage and conversion devices and systems. Energy Environ Sci, 2014, 7: 2101-2122

3 Zhou G, Li F, Cheng HM. Progress in flexible lithium batteries and future prospects. Energy Environ Sci, 2014, 7: 1307-1338

4 Liu Z, Li H, Zhu M, et al. Towards wearable electronic devices: A quasi-solid-state aqueous lithium-ion battery with outstanding stability, flexibility, safety and breathability. Nano Energy, 2018, 44: 164-173

5 Peng X, Peng L, Wu C, et al. Two dimensional nanomaterials for flexible supercapacitors. Chem Soc Rev, 2014, 43: 3303-3323

6 Shao Y, El-Kady MF, Wang LJ, et al. Graphene-based materials for flexible supercapacitors. Chem Soc Rev, 2015, 44: 3639-3665

7 Shown I, Ganguly A, Chen LC, et al. Conducting polymer-based flexible supercapacitor. Energy Sci Eng, 2015, 3: 2-26

8 Zhang $\mathrm{C}, \mathrm{Lv} \mathrm{W}$, Tao Y, et al. Towards superior volumetric performance: Design and preparation of novel carbon materials for energy storage. Energy Environ Sci, 2015, 8: 1390-1403

9 Zhang L, Han D, Tao Y, et al. Dense organic molecules/graphene network anodes with superior volumetric and areal performance for asymmetric supercapacitors. J Mater Chem A, 2020, 8: 461-469

10 Zhang LL, Zhao XS. Carbon-based materials as supercapacitor electrodes. Chem Soc Rev, 2009, 38: 2520-2531

11 Wei W, Cui X, Chen W, et al. Manganese oxide-based materials as electrochemical supercapacitor electrodes. Chem Soc Rev, 2011, 40: 1697-1721

12 Snook GA, Kao P, Best AS. Conducting-polymer-based supercapacitor devices and electrodes. J Power Sources, 2011, 196: 1-12

13 Kumar NA, Choi HJ, Shin YR, et al. Polyaniline-grafted reduced graphene oxide for efficient electrochemical supercapacitors. ACS Nano, 2012, 6: 1715-1723

14 Sha $\mathrm{C}, \mathrm{Lu} \mathrm{B}, \mathrm{Mao} \mathrm{H}$, et al. 3D ternary nanocomposites of molybdenum disulfide/polyaniline/reduced graphene oxide aerogel for high performance supercapacitors. Carbon, 2016, 99: 26-34

15 Fan W, Zhang C, Tjiu WW, et al. Graphene-wrapped polyaniline hollow spheres as novel hybrid electrode materials for supercapacitor applications. ACS Appl Mater Interfaces, 2013, 5: 33823391

16 Zhang J, Jiang J, Li H, et al. A high-performance asymmetric supercapacitor fabricated with graphene-based electrodes. Energy Environ Sci, 2011, 4: 4009-4015

17 Wang Y, Tang S, Vongehr S, et al. High-performance flexible solid-state carbon cloth supercapacitors based on highly processible $\mathrm{N}$-graphene doped polyacrylic acid/polyaniline composites. Sci Rep, 2016, 6: 12883

18 Hu N, Zhang L, Yang C, et al. Three-dimensional skeleton net- 
works of graphene wrapped polyaniline nanofibers: An excellent structure for high-performance flexible solid-state supercapacitors. Sci Rep, 2016, 6: 19777

19 Liu P, Yan J, Gao X, et al. Construction of layer-by-layer sandwiched graphene/polyaniline nanorods/carbon nanotubes heterostructures for high performance supercapacitors. Electrochim Acta, 2018, 272: 77-87

$20 \mathrm{Xu} \mathrm{D}, \mathrm{Xu} \mathrm{Q}$, Wang $\mathrm{K}$, et al. Fabrication of free-standing hierarchical carbon nanofiber/graphene oxide/polyaniline films for supercapacitors. ACS Appl Mater Interfaces, 2014, 6: 200-209

21 Wang Y, Yang X, Qiu L, et al. Revisiting the capacitance of polyaniline by using graphene hydrogel films as a substrate: The importance of nano-architecturing. Energy Environ Sci, 2013, 6: $477-481$

$22 \mathrm{Wu}$ J, Zhang Q, Zhou A, et al. Phase-separated polyaniline/graphene composite electrodes for high-rate electrochemical supercapacitors. Adv Mater, 2016, 28: 10211-10216

23 Chen $\mathrm{K}$, Chen $\mathrm{L}$, Chen $\mathrm{Y}$, et al. Three-dimensional porous graphene-based composite materials: Electrochemical synthesis and application. J Mater Chem, 2012, 22: 20968-20976

24 Armes SP, Miller JF. Optimum reaction conditions for the polymerization of aniline in aqueous solution by ammonium persulphate. Synth Met, 1988, 22: 385-393

25 Wang X, Ding Y, Chen F, et al. Hierarchical porous N-doped graphene monoliths for flexible solid-state supercapacitors with excellent cycle stability. ACS Appl Energy Mater, 2018, 1: 50245032

26 Meng C, Liu C, Chen L, et al. Highly flexible and all-solid-state paperlike polymer supercapacitors. Nano Lett, 2010, 10: 4025-4031

27 Zhang L, Shi G. Preparation of highly conductive graphene hydrogels for fabricating supercapacitors with high rate capability. J Phys Chem C, 2011, 115: 17206-17212

28 Bai H, Li C, Shi G. Functional composite materials based on chemically converted graphene. Adv Mater, 2011, 23: 1089-1115

$29 \mathrm{Xu} \mathrm{Z}$, Gao C. Graphene in macroscopic order: Liquid crystals and wet-spun fibers. Acc Chem Res, 2014, 47: 1267-1276

$30 \mathrm{Xu} \mathrm{Z}$, Gao C. Aqueous liquid crystals of graphene oxide. ACS Nano, 2011, 5: 2908-2915

$31 \mathrm{Xu} \mathrm{Z}$, Gao C. Graphene chiral liquid crystals and macroscopic assembled fibres. Nat Commun, 2011, 2: 571

32 Barman T, Pal AR. Contradictory ageing behaviour and optical property of iodine doped and $\mathrm{H}_{2} \mathrm{SO}_{4}$ doped pulsed DC plasma polymerized aniline thin films. Solid State Sci, 2013, 24: 71-78

33 Tang L, Wang Y, Li Y, et al. Preparation, structure, and electrochemical properties of reduced graphene sheet films. Adv Funct Mater, 2009, 19: 2782-2789

34 Park S, An J, Potts JR, et al. Hydrazine-reduction of graphite- and graphene oxide. Carbon, 2011, 49: 3019-3023

35 Pei S, Zhao J, Du J, et al. Direct reduction of graphene oxide films into highly conductive and flexible graphene films by hydrohalic acids. Carbon, 2010, 48: 4466-4474

36 Zhang Q, Zhou A, Wang J, et al. Degradation-induced capacitance: A new insight into the superior capacitive performance of polyaniline/graphene composites. Energy Environ Sci, 2017, 10: 23722382

37 Yang C, Zhang L, Hu N, et al. Densely-packed graphene/conducting polymer nanoparticle papers for high-volumetric-performance flexible all-solid-state supercapacitors. Appl Surf Sci, 2016, 379: 206-212

38 Li K, Liu J, Huang Y, et al. Integration of ultrathin graphene/ polyaniline composite nanosheets with a robust 3D graphene framework for highly flexible all-solid-state supercapacitors with superior energy density and exceptional cycling stability. J Mater Chem A, 2017, 5: 5466-5474

39 Hong X, Zhang B, Murphy E, et al. Three-dimensional reduced graphene oxide/polyaniline nanocomposite film prepared by diffusion driven layer-by-layer assembly for high-performance supercapacitors. J Power Sources, 2017, 343: 60-66

$40 \mathrm{Xu} \mathrm{Y,} \mathrm{Tao} \mathrm{Y,} \mathrm{Zheng} \mathrm{X,} \mathrm{et} \mathrm{al.} \mathrm{A} \mathrm{metal-free} \mathrm{supercapacitor} \mathrm{electrode}$ material with a record high volumetric capacitance over $800 \mathrm{~F} \mathrm{~cm}^{-3}$ . Adv Mater, 2015, 27: 8082-8087

41 Lu X, Dou H, Yang S, et al. Fabrication and electrochemical capacitance of hierarchical graphene/polyaniline/carbon nanotube ternary composite film. Electrochim Acta, 2011, 56: 9224-9232

42 Pedrós J, Boscá A, Martínez J, et al. Polyaniline nanofiber sponge filled graphene foam as high gravimetric and volumetric capacitance electrode. J Power Sources, 2016, 317: 35-42

43 Wang DW, Li F, Zhao J, et al. Fabrication of graphene/polyaniline composite paper via in situ anodic electropolymerization for highperformance flexible electrode. ACS Nano, 2009, 3: 1745-1752

44 Wang S, Ma L, Gan M, et al. Free-standing 3D graphene/polyaniline composite film electrodes for high-performance supercapacitors. J Power Sources, 2015, 299: 347-355

$45 \mathrm{Wu} \mathrm{Q}, \mathrm{Xu} \mathrm{Y,} \mathrm{Yao} \mathrm{Z,} \mathrm{et} \mathrm{al.} \mathrm{Supercapacitors} \mathrm{based} \mathrm{on} \mathrm{flexible} \mathrm{gra-}$ phene/polyaniline nanofiber composite films. ACS Nano, 2010, 4: 1963-1970

46 Yao B, Yuan L, Xiao X, et al. Paper-based solid-state supercapacitors with pencil-drawing graphite/polyaniline networks hybrid electrodes. Nano Energy, 2013, 2: 1071-1078

47 Gao W, Singh N, Song L, et al. Direct laser writing of microsupercapacitors on hydrated graphite oxide films. Nat Nanotech, 2011, 6: 496-500

48 Yang S, Song X, Zhang $\mathrm{P}$, et al. A $\mathrm{MnOOH} /$ nitrogen-doped graphene hybrid nanowires sandwich film for flexible all-solid-state supercapacitors. J Mater Chem A, 2015, 3: 6136-6145

49 Yang C, Zhang L, Hu N, et al. Reduced graphene oxide/polypyrrole nanotube papers for flexible all-solid-state supercapacitors with excellent rate capability and high energy density. J Power Sources, 2016, 302: 39-45

50 Ma W, Chen S, Yang S, et al. Flexible all-solid-state asymmetric supercapacitor based on transition metal oxide nanorods/reduced graphene oxide hybrid fibers with high energy density. Carbon, 2017, 113: 151-158

51 Chen S, Wang L, Huang $\mathrm{M}$, et al. Reduced graphene oxide $/ \mathrm{Mn}_{3} \mathrm{O}_{4}$ nanocrystals hybrid fiber for flexible all-solid-state supercapacitor with excellent volumetric energy density. Electrochim Acta, 2017, 242: $10-18$

52 Yang C, Zhang L, Hu N, et al. High-performance flexible all-solidstate supercapacitors based on densely-packed graphene/polypyrrole nanoparticle papers. Appl Surf Sci, 2016, 387: 666-673

53 Papageorgiou DG, Kinloch IA, Young RJ. Mechanical properties of graphene and graphene-based nanocomposites. Prog Mater Sci, 2017, 90: 75-127

54 Mittal G, Dhand V, Rhee KY, et al. A review on carbon nanotubes and graphene as fillers in reinforced polymer nanocomposites. J Industrial Eng Chem, 2015, 21: 11-25

Acknowledgements This work was financially supported by the National Natural Science Foundation of China (21774104 and 21975210), and the Natural Science Foundation of Fujian Province (2018J06015). 
Author contributions Bai H conceived the project. Wang J, Su W, Zhang $\mathrm{J}$ and Zhou A performed the experiments. Zhang $\mathrm{T}$ and Bai $\mathrm{H}$ supervised the project. Wang J, Bai $\mathrm{H}$ and Zhang $\mathrm{T}$ wrote the manuscript. All authors contributed to the discussion and preparation of the manuscript. The final version of the manuscript was approved by all authors.

Conflict of interest The authors declare no conflict of interest.

Supplementary information Supporting data are available in the online version of the paper.

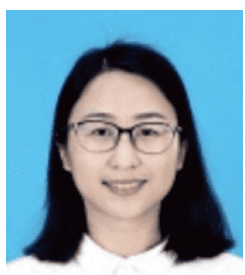

Jingjing Wang obtained her MSc from the Southeast University. She is currently a $\mathrm{PhD}$ student at the College of Materials, Xiamen University, under the supervision of Prof. Hua Bai and Prof. Tong-Yi Zhang. Her research interests focus on conducting polymers and supercapacitors.

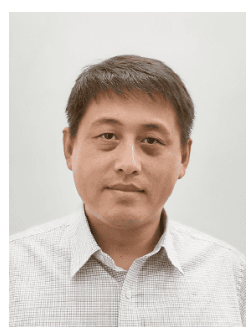

Hua Bai received his $\mathrm{BSc}$ and $\mathrm{PhD}$ degrees from the Department of Chemistry, Tsinghua University in 2004 and 2009, respectively. From 2009 to 2011, he worked in the Department of Chemical Engineering, Tsinghua University, as a postdoctoral researcher. He is currently an professor at the College of Materials, Xiamen Univeristy. His research focuses on the energy conversion and storage, including developing new materials for supercapacitors, lithium ion batteries and solar cells.

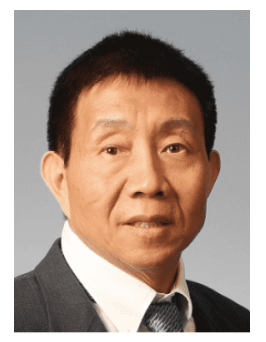

Tong-Yi Zhang earned his Master degree in 1982 and $\mathrm{PhD}$ in 1985, majored in materials physics, from the University of Science and Technology Beijing, China. He is the Founding Dean of the Materials Genome Institute, Shanghai University. He is committed to promoting and fostering the developments of materials genome initiative (MGI) and materials/mechanics informatics, which integrate data science, artificial intelligence, and machine learning with materials science and engineering, and mechanics science and engineering, respectively.

\section{柔性聚苯胺电极体积电容的提高：溶液浇铸法以 及还原氧化石墨烯的影响}

\author{
王菁菁 ${ }^{1}$, 苏炜华 $^{1}$, 张金梦 $^{1}$, 周安安 ${ }^{1}$, 白华 $^{1^{*}}$, 张统一 $^{2^{*}}$
}

摘要 高体积比电容是柔性超级电容器的关键. 在本文中, 我们设 计并制备了一种具有超高体积比电容和良好倍率性能的柔性复合 电极. 该膜电极以无孔且致密的聚苯胺作为基质, 少量的还原氧化 石墨烯片作为填料，通过一种新开发的溶液浇铸法制备而成. 用于 浇铸的溶液是由聚苯胺和氧化石墨烯的混合溶液自组装制备得到. 关于还原氧化石墨烯作用的系统研究结果表明, 还原氧化石墨烯 既是导电填料又是扩散屏障. 通过优化还原氧化石墨烯的含量, 电 极在 $2.4 \mathrm{~A} \mathrm{~cm}^{-3}$ 下体积比电容高达 $1354 \mathrm{~F} \mathrm{~cm}^{-3}$; 同时由于聚苯胺具 有良好的离子电导率, 电极也表现出良好的倍率性能. 本工作为柔 性超级电容器提供了一种高性能的电极, 同时溶液浇铸法有望应 用于其他有机电极材料的制备. 